\title{
ANÁLISE DOS RESÍDUOS MADEIREIROS GERADOS NAS MARCENARIAS DO MUNICÍPIO DE VIÇOSA - MINAS GERAIS ${ }^{1}$
}

\author{
Zaíra Morais dos Santos Hurtado de Mendoza², Wescley Viana Evangelista², Solange de Oliveira \\ Araújo² $^{2}$ Celso Coelho de Souza², Fabiana David Leite Ribeiro² e José de Castro Silva ${ }^{3}$

\begin{abstract}
RESUMO - O objetivo deste trabalho foi analisar os resíduos gerados nas marcenarias do município de Viçosa, Minas Gerais, e propor ações potencializadoras para o seu melhor aproveitamento. Foram coletados dados sobre os resíduos de madeira gerados em 17 marcenarias da cidade, por intermédio da aplicação de questionários. Os resultados mostraram que a serragem, os sarrafos, as maravalhas e os cavacos foram os principais tipos de resíduos produzidos, sendo a serragem o resíduo mais abundante. As máquinas que mais geraram resíduos foram a desengrossadeira e a desempenadeira. A maioria das marcenarias doava ou vendia os resíduos produzidos. Constatou-se que não havia preocupação por parte dos proprietários das marcenarias quanto à agregação de valor ao resíduo gerado e quais os danos ambientais que estes poderiam ocasionar. A partir dos resultados, propuseram-se as seguintes medidas de melhor aproveitamento dos resíduos gerados: geração de energia através da queima direta de resíduos oriundos da madeira sólida, fabricação de briquetes, fertilizantes e de "pequenos objetos de madeira" (POM).
\end{abstract}

Palavras-chave: Viçosa-Minas Gerais, Marcenarias e Resíduos de Madeira.

\section{AN ANALYSIS OF THE WOOD RESIDUES GENERATED BY CARPENTRY SHOPS IN VIÇOSA, STATE OF MINAS GERAIS}

\begin{abstract}
The objectives of this work were to analyze the residues produced by carpentry shops in Viçosa and to propose actions that would lead to their better use. For this, data were gathered from questionnaires sent to 17 shops in town. The results showed that sawdust, wood strips, planner shavings and chips are the more common type of residues, sawdust being the most abundant. The machines that produced most of the waste were the planners and the jointers. Most of the shops either donate or sell their waste. It was found that the owners showed no regard to increasing the value of the residue or to environmental damage they may cause. Based on the information collected, the following measures are indicated for waste utilization: generation of energy through direct burning of solid wood residues, production of briquet and fertilizers and production of the so called small wooden articles.
\end{abstract}

Keywords: Viçosa - Minas Gerais - Brazil, Carpentry shops and Wood Residues.

\section{INTRODUÇÃO}

Independente do tipo de indústria madeireira, a geração de resíduos é consequência direta do processamento primário ou secundário da madeira sólida ou do processamento dos painéis reconstituídos, como compensados, aglomerados e Medium Density Fiberboard (MDFs). Segundo IBQP (2002), de acordo com suas características morfológicas os resíduos dessas indústrias são classificados como cavacos (partículas com dimensões máximas de 50 x 20 mm, em geral provenientes do uso de picadores), maravalhas (resíduo com menos de 2,5 mm), serragem (partículas de madeira com dimensões entre 0,5 a $2,5 \mathrm{~mm}$, provenientes do uso de serras) e, por fim, o pó (resíduos menores que 0,5 mm). Existe também o resíduo de madeira denominado sarrafo que, segundo SENAI

\footnotetext{
${ }^{1}$ Recebido em 27.12.2007 e aceito para publicação em 02.03.2010.

${ }^{2}$ Programa de Pós-Graduação em Ciência Florestal pela Universidade Federal de Viçosa, MG, Brasil. E-mail: <zaira@ufmt.br>, <wescleyviana@yahoo.com.br>, <solangeeoa@yahoo.com.br>, <celsoenf01@yahoo.com.br>e <ffabianaribeiro@yahoo.com.br>.

${ }^{3}$ Professor Adjunto do Departamento de Engenharia Florestal da Universidade Federal de Viçosa,Viçosa, MG. E-mail: <jcastro@ufv.br> .
} 
(1994), corresponde a uma peça de madeira com topo de dimensões reduzidas, porém sem diferença marcante entre a largura e a espessura.

No Brasil, a indústria de móveis sob encomenda se caracteriza, segundo Gorini (1998), por serem predominantemente micro e pequenas empresas, em geral marcenarias, tendo como matéria-prima básica os painéis reconstituídos conjugados com madeiras nativas, cujo produto final se destina predominantemente ao mercado doméstico ou local. Silva et al. (2002), avaliando o perfil de trabalhadores e das condições de trabalho em marcenarias no Município de Viçosa, MG, verificaram que esse perfil de indústria observado na cidade é também semelhante ao encontrado no restante do país. Esses mesmos autores observaram também que os principais resíduos madeireiros produzidos nas marcenarias do município são na forma de serragem, sendo acondicionados, em maior parte das marcenarias, nos próprios galpões de trabalho. Os referidos autores consideraram esse armazenamento com risco de incêndios, considerando que a maioria dos marceneiros fazia uso do fumo, inclusive durante o trabalho, o que coloca em risco a segurança dos trabalhadores e da própria vizinhança das marcenarias.

Considerando a falta de usos potencializadores para os resíduos gerados nas marcenarias de Viçosa, este trabalho teve como objetivos analisar a produção de resíduos e propor ações potencializadoras para o seu melhor aproveitamento.

\section{MATERIAL E MÉTODOS}

Para a avaliação da geração dos resíduos madeireiros produzidos nas marcenarias do Município de Viçosa, MG, foi realizada a coleta de dados em 17 marcenarias da cidade, correspondendo a uma amostragem equivalente a $53 \%$ do total. O critério de seleção dessas marcenarias foi com base em seus registros na Câmara de Dirigentes Lojistas (CDL) do município. Todas as empresas registradas no CDL foram visitadas e tiveram a avaliação dos resíduos gerados. As demais marcenarias do município não foram avaliadas em razão de o proprietário não aceitar disponibilizar os dados dos resíduos gerados pela sua companhia. As marcenarias avaliadas em Viçosa são classificadas, segundo SEBRAE (2002), como microempresas, por possuírem até, no máximo, 10 empregados. A obtenção dos dados foi feita através da aplicação de questionário, conforme pode ser visto na Figura 1, durante o período de junho a julho de 2006.

O questionário aplicado consistiu de cinco partes. Na primeira foram coletados os dados cadastrais da marcenaria e do entrevistado; na segunda os dados sobre os tipos de matérias-primas utilizados, bem como a sua origem; na terceira, os dados sobre os equipamentos utilizados nas marcenarias; na quarta, sobre o modelo de produção dos móveis; e na quinta e última parte, coletados os dados a respeito dos tipos de resíduos, dos equipamentos geradores e sobre o seu destino final. Justificou-se a inclusão das partes segunda a quarta nesse questionário para melhor caracterização das marcenarias do Município de Viçosa, visando, principalmente, ao conhecimento das matérias-primas utilizadas, maquinário presente e produção mensal de móveis ou outros produtos, para melhor compressão e interpretação dos dados sobre a geração dos resíduos madeireiros.

O questionário foi aplicado em forma de entrevista individual com o proprietário ou responsável pela marcenaria, no próprio local de trabalho, onde as perguntas foram rápidas e objetivas, de maneira a propiciar boa aceitação e compreensão pelos entrevistados. Para a quantificação dos resíduos foram coletados, semanalmente, todos os resíduos gerados pelas marcenarias.. Os resíduos foram quantificados em $\mathrm{m}^{3}$, utilizando-se uma caixa de madeira como gabarito, cujas dimensões eram de 1 m x 1 m x 1 m. A análise e interpretação dos dados foram realizadas através de análise estatística descritiva, em planilhas do Excel.

\section{RESULTADOS}

Com relação às matérias-primas utilizadas, todas as marcenarias obtêm seu material na forma de painéis reconstituídos ou madeira serrada através de terceiros ou fornecedores específicos. Observou-se que nenhuma marcenaria possuía plantios próprios para seu suprimento de madeira. Predominou-se o uso de painéis reconstituídos $(57,69 \%)$, seguidos das pranchas de madeira sólida e, em menor porcentagem, o uso conjugado dos painéis reconstituídos mais as pranchas de madeira sólida, conforme pode ser visto na Figura 2a. A origem das matérias-primas, segundo os entrevistados, foi predominantemente da Região Sudeste do Brasil, como mostrado na Figura 2b. 
a) Tipo de matéria-prima

a) Type of raw material

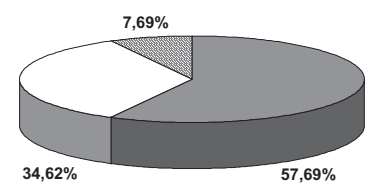

$\square$ Paineis $\square$ Pranchas 圆Conjugado b) Origem da matéria-prima

b) Origin of the raw material

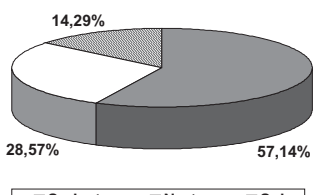

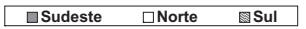

Figura 2 - Matéria-prima utilizada pelas marcenarias. Figure 2 - Raw material used by shops.

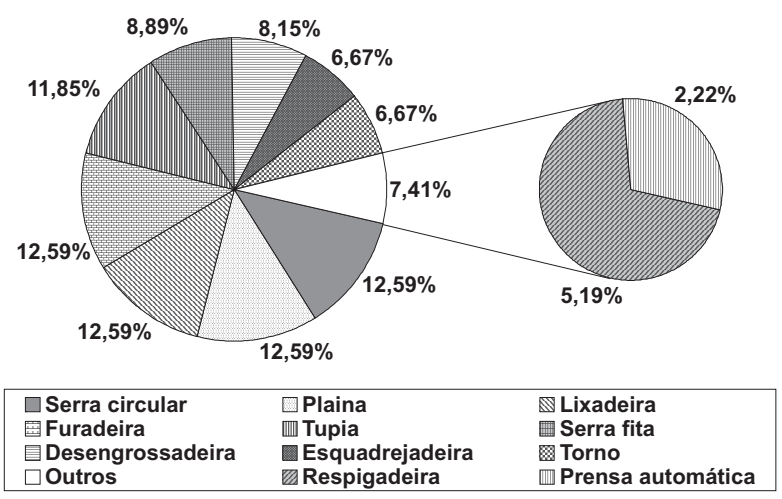

Figura 3 - Porcentagem média das principais máquinas. Figure 3 - Mean percentage of the main machines.

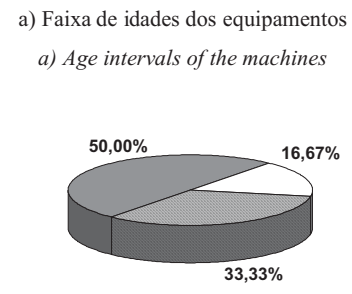

$\square$ De 0 a $20 \square$ De 21 a $40 \square$ Acima de 40 b) Periodicidade na manutenção

b) Periodicity of maintenance
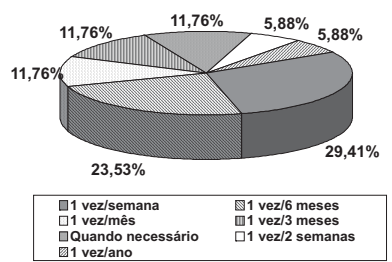

Figura 4 - Idade das máquinas e periodicidade de manutenção. Figure 4-Age of the machines and periodicity of maintenance.

a) Principais artigos fabricados

a) Principal manufactured articles

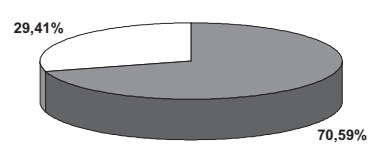

पMóveis $\square$ Artigos $\mathrm{p} /$ carpinteria b) Volume de produção

b) Volume of production

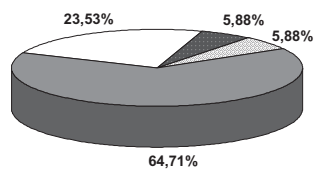

$\square 1$ a $30 \quad \square 31$ a $60 \quad \square 61$ a $90 \quad \square 91$ a 150

Figura 5 - Principais indicadores de produção nas marcenarias visitadas.

Figure 5-Main production indicators for the shops visited.

a) Principais tipos de resíduos gerados a) Main residue types

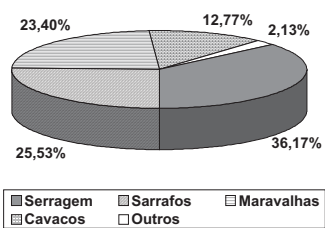

Figura 6 - Principais tipos de resíduos e suas máquinas geradoras. Figure 6-Main residue types and machines producing such types.

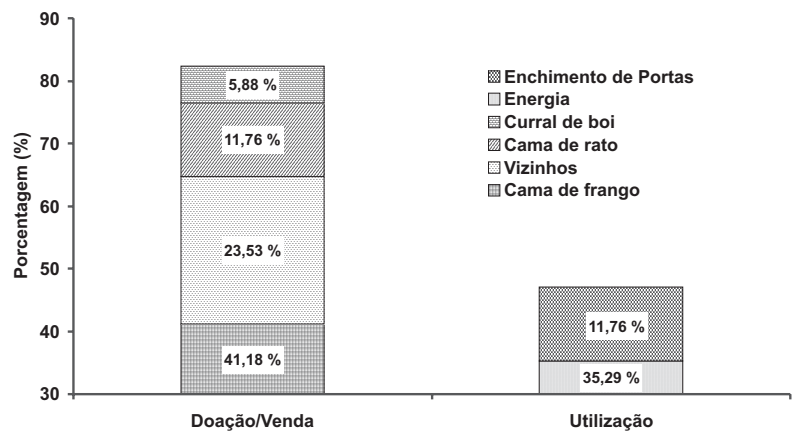

Figura 7 - Principais usos dos resíduos gerados. Figure 7-Most common residue uses.

de resíduos, de origem não madeireira, também foram registrados como produzidos pelas marcenarias de Viçosa, como fórmica, lã, linha e espuma, totalizando 2,13\% do total. Segundo os entrevistados, as máquinas que mais geraram resíduos foram a desengrossadeira e a desempenadeira (Figura 5b).

Na Figura 7 são apresentados os principais destinos dos resíduos gerados pelas marcenarias avaliadas. Constatou-se que a maioria das empresas doava ou vendia seus resíduos, os quais se destinavam à forração para criação de frangos (41,18\%), consumo doméstico (23,53\%), forração para criação de ratos $(11,76 \%)$ e para limpeza de curral (5,88\%). Porém, algumas empresas (47,05\%) também utilizavam os resíduos para fins energéticos (35,29\%) e enchimento de portas produzidas pela própria marcenaria $(11,76 \%)$. Com base nas entrevistas com os proprietários ou responsáveis pelas marcenarias visitadas, constatou-se que eles não tinham preocupação quanto à maior agregação de valor ao resíduo gerado ou quanto aos danos ambientais que estes resíduos poderiam causar, caso fossem descartados de maneira incorreta. 


\section{DISCUSSÃO}

O conhecimento dos resíduos gerados por uma empresa tem importância tanto econômica quanto ambiental, pois se espera maior retorno econômico durante todo o processo produtivo e menor dano ambiental. Esses dois aspectos podem ser amplamente resolvidos através do aproveitamento máximo dos resíduos gerados durante toda a cadeia produtiva, como pode acontecer na indústria madeireira.

Conforme descrito por Silva et al. (2002), as marcenarias do município de Viçosa são caracterizadas pela fabricação de móveis sob encomenda, sendo a produção praticamente artesanal, com o auxílio de algumas máquinas utilizadas para agilizar e facilitar o processo de fabricação. Os móveis fabricados destinavam-se, principalmente, ao consumo doméstico no próprio município e a outras cidades da região. Tendo em vista esses aspectos, é de suma importância ter-se também a preocupação com o destino final dos resíduos, gerados durante a produção dos móveis. Segundo Cassilha et al. (2004), o aproveitamento de resíduos da madeira sólida poderia ser em granjas, como forração para a criação de animais, e também na agricultura, para auxiliar na retenção de umidade do solo, uma vez que a maioria desses resíduos não é tóxica ao meio ambiente, pois a madeira é material naturalmente biodegradável. Para os painéis reconstituídos, o aproveitamento de resíduo estava mais limitado, devido à presença de componentes tóxicos durante o seu processo de fabricação, como adesivos, aditivos químicos e produtos de acabamento superficial.

O destino final dos resíduos de madeira nas marcenarias estava inserido em algumas recomendações do seu aproveitamento, descritos na literatura. O uso dos resíduos na forração para criação de frangos já é um uso potencial, sendo doado e vendido pelos donos das marcenarias.

Com base nos resultados, propõem-se as seguintes medidas potencializadoras do melhor aproveitamento dos resíduos gerados nas marcenarias:

- Geração de energia através da queima direta de resíduos oriundos da madeira sólida.

- Fabricação de briquetes e fertilizantes.

- Fabricação de "pequenos objetos de madeira" (POM), que são definidos como pequenas peças confeccionadas de modo industrial ou artesanal que podem ser manuseadas ou conduzidas facilmente com as mãos. Segundo Sternadt (2002), os chamados “pequenos objetos de madeira”, também conhecidos pela sigla POM, têm grande importância no cotidiano das pessoas. Estão presentes nos mais diversos tipos: artigos domésticos de caráter utilitário e decorativo, complementos de outros materiais, artigos de uso pessoal, brinquedos e artigos esportivos.

Sugere-se que, em cada aproveitamento proposto, seja realizado um estudo mais detalhado para cada utilização, visando ao maior retorno econômico para as marcenarias e também menor risco ambiental, devido à geração de resíduos nocivos ao meio ambiente.

\section{CONCLUSÕES}

De acordo com os resultados, concluiu-se que:

- A produção de resíduos nas marcenarias de Viçosa é pequena ao longo do período de um mês.

- Há novos usos potenciais para o aproveitamento dos resíduos produzidos pelas marcenarias de Viçosa, sobretudo referente à produção de novos produtos, como briquetes e fertilizantes e também para a fabricação de pequenos objetos de madeira.

- Necessita-se de estudos mais detalhados sobre o aproveitamento de resíduos dentro das empresas estudadas, para que elas possam agregar valor aos seus produtos, buscando maiores retornos econômicos.

\section{REFERÊNCIAS}

CASSILHA, A. C. et al. Indústria moveleira e resíduos sólidos: considerações para o equilíbrio ambiental. Revista Educação \&

Tecnologia, v.8, p.209-228, 2004.

GORINI, A. P. F. Panorama do setor moveleiro no Brasil, com ênfase na competitividade externa a partir do desenvolvimento da cadeia industrial de produtos sólidos de madeira. Brasília: BNDES, 1998. 47p.

INSTITUTO BRASILEIRO DE QUALIDADE E PRODUTIVIDADE DO PARANÁ - IBQP. Análise da competitividade da cadeia produtiva da madeira no estado do Paraná. Curitiba. 2002. 345f. (Relatório Final. IBQP).

Revista Árvore, Viçosa-MG, v.34, n.4, p.755-760, 2010 
SERVIÇO BRASILEIRO DE APOIO ÀS MICRO E PEQUENAS EMPRESAS - SEBRAE. A micro e pequena empresa no Brasil. Brasília: Ministério do Trabalho, 2002. Disponível em $<200.252 .248 .100 /$ site/na/conmpe.nsf $>$. Acesso em 21 ago. 2006.

SENAI/RS. Glossário mobiliário madeira. Bento Gonçalves: CETEMO-NIT/ MM, 1994. 173p.
SILVA, K. R.; SOUZA, A. P.; MINETTI, L. J. Avaliação do perfil de trabalhadores e das condições de trabalho em marcenarias no município de Viçosa-MG. Revista Árvore, v.26, n.6, p.769-775, 2002.

Sternadt, G. H. Pequenos objetos de madeira - POM, compostagem de serragem de madeira. Brasília: LPF, 2002. 29p. 